J. Austral. Math. Soc. 20 (Series A) (1975), 146-158.

\title{
RECURSIVE DENSITY TYPES AND NERODE EXTENSIONS OF ARITHMETIC
}

\author{
P. ACZEL \\ (Received 20 December 1972) \\ Communicated by J. N. Crossley
}

\section{Introduction}

The notion of a recursive density type (R.D.T.) was introduced by Medvedev and developed by Pavlova (1961). More recently the algebra of R.D.T.'s was initiated by Gonshor and Rice (1969). The R.D.T.'s are equivalence classes of sets of integers, similar in many respects to the R.E.T.'s. They may both be thought of as effective analogues of the cardinal numbers. While the equivalence relation for R.E.T.'s is defined in terms of partial recursive functions, that for R.D.T.'s may be characterized in terms of recursively bounded partial functions (see 4.22a).

In Gronshor and Rice (1969) addition and multiplication of R.D.T.'s are defined and some of their properties are found. In particular a subset $\Lambda_{s}$ of the R.D.T.'s is defined such that the following cancellation law holds; $x+y=x+z$ implies $y=z$ for $x \in \Lambda_{s}$ where $y, z$ are arbitrary R.D.T.'s. This led them to conjecture that there is an extension theory for R.D.T.'s analogous to Nerode's theory for the R.E.T.'s (see Nerode (1961)) and that certain subclasses such as $\Lambda_{s}$ would have properties similar to $\Delta$, the set of isols.

The aim of this paper is to verify this conjecture. We shall show that there is a natural procedure to extend arbitrary relations on $\omega=\{0,1, \cdots\}$ to relations on R.D.T.'s and that a large class of functions on $\omega$ extend to functions on R.D.T.'s. A generalization of the above cancellation law for addition will be seen to apply to a set $\Gamma \supseteq \Delta_{s}$.

In order to put the above results in a general framework we define the notion of a Nerode extension of arithmetic. Roughly speaking, a Nerode extension is a relational system, extending a relational system with domain $\omega$, whose universal properties may be characterized as in Theorem 11.1 of Nerode (1961). Thus the main result of Nerode (1961) is that a certain relational system on $\Delta$ is a Nerode extension. The main result of this paper is that there is a relational systme on $\Gamma$ that is a Nerode extension. 
In the first part of the paper we define and investigate Nerode extensions and give some examples. In the second part we introduce the R.D.T.'s and prove the main results. The method used to extend relations and functions on $\omega$ to the R.D.T.'s is by a characterization of the R.D.T.'s as a homomorphic image of a previously defined Nerode extension. This characterization has led to simpler proofs of many of the results.

\section{Part I}

1.1. Let ${ }^{k} A$ denote the $k$-fold cartesian product of the set $A$. If $x \in{ }^{k} A$ then $x=\left(x_{0}, \cdots, x_{k-1}\right)$.

If $h: I \rightarrow \omega$ where $I$ is a proper subset of $\{0, \cdots, k-1\}$ and $R \subseteq{ }^{k} \omega$, the $k$-specification $S_{h} R$ of $R$ is the relation obtained from $R$ by substituting the integer $h(i)$ at the $i$-th argument place for each $i \in I$. More precisely, if $J=\{0, \cdots, k-1\}-I=\{j(0)<\cdots<j(t-1)\}$ then $S_{h} R=\left\{x \in{ }^{t} \omega \mid h^{*}(x) \in R\right\}$ where if $x \in^{t} \omega$ then $h^{*}(x)=y$, where $y_{i}=h(i)$ for $i \in I$ and $y_{j(i)}=x_{i}$ for $i<t$.

$R \subseteq{ }^{k} \omega$ is totally unbounded if for all $x \in{ }^{k} \omega$ there is a $y \in R$ such that $x \leqq y$ (i.e. $x_{i} \leqq y_{i}$ for $i<k$ ). If $R, S \subseteq{ }^{k} \omega$ let $R \subseteq{ }_{e} S$ if $R-S$ is not totally unbounded. $R$ is eventual if ${ }^{k} \omega \subseteq \subseteq_{e} R$.

The following is needed in the proof of Lemma 1.4.

Lemma. $R \subseteq{ }^{k} \omega$ is co-finite if and only if every $k$-specification $S_{h} R$ is eventual.

1.2. Let $\mathscr{L}$ be the full first order language for arithmetic, i.e. there is a function symbol $f$ for each function on $\omega$ and a relation symbol $\underline{R}$ for each relation $R$ on $\omega$. If $\phi$ is a quantifier free formula of $\mathscr{L}$ in conjunctive normal form then $\phi^{\prime}$ is a Horn reduct of $\phi$ if $\phi^{\prime}$ can be obtained from $\phi$ by striking out all but one unnegated atomic formula in each conjunct with at least two occurrences of unnegated atomic formulae.

If $\mathscr{F}^{n}$ is a set of $n$-ary functions on $\omega$ for $n=1,2, \cdots$ and $\mathscr{F}=\cup\left\{\mathscr{F}^{n} \mid 0\right.$ $<n<\omega\}$, let $\mathscr{L}(\mathscr{F})$ be the sublanguage of $\mathscr{L}$ with symbols $f$ for $f \in \mathscr{F}$ and $\underline{R}$ for $R \in \mathscr{R}(\mathscr{F})=\cup\left\{\mathscr{R}^{n}(\mathscr{F}) \mid 0<n<\omega\right\}$, where $R \in \mathscr{R}^{n}(\mathscr{F})$ if and only if $R=\left\{x \in{ }^{n} \omega \mid f(x)=g(x)\right\}$ for some $f, g \in \mathscr{F}^{n}$.

$\mathscr{F}$ will be called a closed system if (1) $u_{i}^{n}, c_{k}^{n} \in \mathscr{F}^{n}$ for $i<n, k \in \omega$, where $u_{i}^{n}(x)=x_{i}, c_{k}^{n}(x)=k$ for $x \in{ }^{n} \omega . \quad$ (2) If $f \in \mathscr{F}^{n}, g^{0}, \cdots, g^{n-1} \in \mathscr{F}^{k}$ then $f \circ\left(g^{0}, \cdots, g^{n-1}\right) \in \mathscr{F}^{k}$ where $f \circ\left(g^{0}, \cdots, g^{n-1}\right)(x)=f\left(g^{0}(x), \cdots, g^{n-1}(x)\right)$ for $x \in{ }^{k} \omega$. (3) If $R, S \in \mathscr{R}^{n}(\mathscr{F})$ then $R \cap S \in \mathscr{R}^{n}(\mathscr{F})$.

The relational system $\mathscr{N}(Q, \mathscr{F})=\left\langle Q, f_{Q}, R_{Q}\right\rangle_{f \in \mathscr{F}, R \in \mathscr{R}(\mathscr{F})}$ is an extension if $\mathscr{F}$ is a closed system, $\omega \subseteq Q$ and $f=f_{Q} \Gamma^{n} \omega, R=R_{Q} \cap^{n} \omega$ for $f \in \mathscr{F}^{n}$, $R \in \mathscr{R}^{n}(\mathscr{F})$. Let $\mathscr{N}(\mathscr{F})=\langle\omega, f, R\rangle_{f \in \mathscr{F}, R \in \mathscr{H}(\mathscr{F})}$. 
1.3. Definition. The extension $\mathscr{N}(Q, \mathscr{F})$ is a Nerode extension if its universal properties may be characterized as follows: If $\phi$ is a quantifier-free formula of $\mathscr{L}(\mathscr{F})$ in conjunctive normal form, with free variables among $v_{0}, \cdots, v_{k-1}$, then $\mathscr{N}(Q, \mathscr{F}) \vDash \forall v_{0} \cdots \forall v_{k-1} \phi$ if and only if (1) $\mathscr{N}(\mathscr{F}) \vDash \forall v_{0} \cdots \forall v_{k-1} \phi$ and (2) for every $k$-specification $S_{h}$ there is a Horn reduct $\phi^{\prime}$ of $\phi$ such that $S_{h}\left\{x \in{ }^{k} \omega \mid \mathscr{N}(\mathscr{F}) \vDash \phi^{\prime}[x]\right\}$ is eventual.

1.4. A proof of the following lemma may be abstracted from Ellentuck (1967).

Lemma. If the extension $\mathscr{N}(Q, \mathscr{F})$ satisfies 1.4.1-1.4.6 then it is a Nerode extension.

1.4.1. $\left(u_{i}^{n}\right)_{Q}(x)=x_{i}$ and $\left(c_{k}^{n}\right)_{Q}(x)=k$ for $x \in{ }^{n} Q$.

1.4.2. If $f \in \mathscr{F}^{n}, g^{0}, \cdots, g^{n-1} \in \mathscr{F}^{k}$ then $\left(f \circ\left(g^{0}, \cdots, g^{n-1}\right)\right)_{Q}=f_{Q} \circ\left(g_{Q}^{0}, \cdots, g_{Q}^{n-1}\right)$.

1.4.3. If $R=\left\{x \in{ }^{n} \omega \mid f(x)=g(x)\right\}$ for $f, g \in \mathscr{F}^{n}$ then $R_{Q}=\left\{x \in{ }^{n} Q \mid f_{Q}(x)=g_{Q}(x)\right\}$.

1.4.4. If $R, S \in \mathscr{R}^{n}(\mathscr{F})$ then $(R \cap S)_{Q}=R_{Q} \cap S_{Q}$.

1.4.5. If $R, S \in \mathscr{R}^{n}(\mathscr{F})$ and $R \subseteq{ }_{e} S$ then $R_{Q^{\infty}}=(R \cap S)_{Q^{\infty}}$ where $R_{Q \infty}=R_{Q} \cap{ }^{n} Q^{\infty}$ for $Q^{\infty}=Q-\omega$.

1.4.6. If $R, R^{0}, \cdots, R^{m-1} \in \mathscr{R}^{n}(\mathscr{F})$ and $R_{Q^{\infty}} \subseteq R_{Q^{\infty}}^{0} \cup \cdots \cup R_{Q^{\infty}}^{m-1}$ then $R \subseteq{ }_{e} R^{i}$ for some $i<m$.

2.1. If $f, g: \omega \rightarrow \omega$ let $f \sim g$ if $\{x \mid f(x)=g(x)\}$ is co-finite. If $\mathscr{F}$ is a closed system let $\mathscr{N}^{\sim}(\mathscr{F})=\mathscr{N}(\mathscr{F} \mid \sim, \mathscr{F})$ where $\mathscr{F} \mid \sim=\left\{f^{\sim} \mid f \in \mathscr{F}^{1}\right\}$ for $f^{\sim}=$ $\left\{g \in^{\omega} \omega \mid f \sim g\right\}$. If $f \in \mathscr{F}^{n}$ let $f_{\mathscr{F} / \sim}:{ }^{n} \mathscr{F}|\sim \rightarrow \mathscr{F}| \sim \operatorname{such}$ that $f_{\mathscr{F} / \sim}\left(f_{0}^{\sim}, \cdots, f_{n-1}^{\sim}\right)=$ $\left(f \circ\left(f_{0}, \cdots, f_{n-1}\right)\right)^{\sim}$. If $R \in \mathscr{R}^{n}(\mathscr{F})$ let $\left(f_{0}^{\sim}, \cdots, f_{n-1}^{\sim}\right) \in R_{\mathscr{F} /}$ if and only if $\left\{x \mid\left(f_{0}(x), \cdots, f_{n-1}(x)\right) \in R\right\}$ is co-finite.

2.2. Identifying $k \in \omega$ with $\left(c_{k}^{1}\right)^{\sim}, \mathscr{N}^{\sim}(\mathscr{F})$ is clearly an extension. Moreover, by routine computations:

2.2.1. $\mathscr{N}^{\sim}(\mathscr{F})$ is an extension satisfying 1.4.1-1.4.4.

2.2.2. If $\mathscr{F}^{1}$ contains only nondecreasing functions then $\mathscr{N}^{\sim}(\mathscr{F})$ satisfies 1.4.5.

Proof. In general if $R, S \in \mathscr{R}^{n}(\mathscr{F})$ then $(R \cap S)_{\mathscr{F} / \sim} \subseteq R_{\mathscr{F} / \sim} \cap S_{\mathscr{F} / \sim}$, for if $f_{0}, \cdots, f_{n-1} \in \mathscr{F}^{1}$ and $\left\{x \mid\left(f_{0}(x), \cdots, f_{n-1}(x)\right) \in R \cap S\right\}$ is co-finite then so are $\left\{x \mid\left(f_{0}(x), \cdots, f_{n \rightarrow 1}(x)\right) \in R\right\}, \quad\left\{x \mid\left(f_{0}(x), \cdots, f_{n-1}(x)\right) \in S\right\} . \quad$ If $\quad R \subseteq_{e} S$ and $\left(f_{0}^{\sim}, \cdots, f_{n-1}^{\sim}\right) \in R_{\mathscr{F} / \sim}$ then $\left\{x \mid\left(f_{0}(x), \cdots, f_{n-1}(x)\right) \in R\right\}$ is co-finite. As $f_{0}^{\sim}, \cdots, f_{n-1}^{\sim} \in \mathscr{F} / \sim \sim^{\infty}$, for every $x \in{ }^{n} \omega$ there is a $k \in \omega$ such that $\forall m \geqq k$ $x \leqq\left(f_{0}(m), \cdots, f_{n-1}(m)\right) \in R$. As $R-S$ is not totally unbounded there is an $x$ such that $y \notin R-S$ for all $y \geqq x$. Hence $\left(f_{0}(m), \cdots, f_{n-1}(m)\right) \in R \cap S$ for all $m \geqq k$, i.e. $\left(f_{0}^{\sim}, \cdots, f_{n-1}^{\sim}\right) \in(R \cap S)_{\mathscr{F} / \sim}$. Hence $(R \cap S)_{\mathscr{F} / \sim}=R_{\mathscr{F} / \sim \infty}$. 
2.3. Definition. $\mathscr{F}$ is good if $\mathscr{F}$ is a closed system such that $\mathscr{F}^{1}$ contains only nondecreasing functions and $\mathscr{F}$ satisfies 2.3 .1 .

2.3.1. If $R, R^{0}, \cdots, R^{m-1} \in \mathscr{R}^{n}(\mathscr{F})$ and $R \oiint_{e} R^{i}$ for all $i<m$ then there are $f_{0}, \cdots, f_{n-1} \in \mathscr{F}^{1}$ such that for each $k \in \omega\left\{x \mid f_{i}(x) \geqq k\right\}$ is co-finite for $i<m$, $\left\{x \mid\left(f_{0}(x), \cdots, f_{n-1}(x)\right) \in R\right\}$ is co-finite and $\left\{x \mid\left(f_{0}(x), \cdots, f_{n-1}(x)\right) \in R^{i}\right\}$ is not co-finite for $i<m$.

2.3.2. Lemma. If $\mathscr{F}$ is good, then $\mathscr{N}^{\sim}(\mathscr{F})$ satisfies $1.4 .1-1.4 .6$ and is hence a Nerode system.

This is an immediate consequence of 2.2.1, 2.2.2 and the observation that 2.3.1 is a restatement of 1.4.6 for $Q=\mathscr{F} / \sim$.

2.4. In this subsection we give some examples of good systems $\mathscr{F}$. Let $\mathscr{F}_{0}$ be the set of functions on $\omega$ that are nondecreasing in exch argument. Let $\mathscr{F}_{r}\left(\mathscr{F}_{c}\right)\left[\mathscr{F}_{r c}\right]$ be the set of recursive (combinatorial) [recursive combinatorial] functions in $\mathscr{F}_{0}$. (See Ellenbuck (1967) or Nerode (1961) for the definition of the combinatorial functions.) Note that $\mathscr{F}_{r c}=\mathscr{F}_{r} \cap \mathscr{F}_{c}$ and that every combinatorial function is in $\mathscr{F}_{0}$. Observe the following

2.4.1. $\mathscr{R}\left(\mathscr{F}_{0}\right)=\mathscr{R}\left(\mathscr{F}_{c}\right)=$ Set of all relations on $\omega$. $\mathscr{R}\left(\mathscr{F}_{r}\right)=\mathscr{R}\left(\mathscr{F}_{r c}\right)=$ Set of recursive relations on $\omega$.

(See for example the first paragraph of $\S 9$ of Nerode (1961).)

2.4.2. Lemma. $\mathscr{F}_{0}, \mathscr{F}_{r}, \mathscr{F}_{c}, \mathscr{F}_{r c}$ are all good.

Proof. The only problem is to show 2.3.1. Let $R, R^{0}, \cdots, R^{m^{-1}} \subseteq{ }^{n} \omega$ such that $R \oiint_{e} R^{i}$ for $i<m$, i.e. each $R-R^{i}$ is totally unbounded. Hence a sequence $\left\langle x^{i} \mid i \in \omega\right\rangle$ of elements of ${ }^{n} \omega$ may be defined such that $x_{j}^{i}<x_{j}^{i+1}$ for all $i \in \omega$ and $j<n$ and if $k \equiv i(\bmod m)$ then $x^{k} \in R-R^{i}$. Hence if $f_{j}(i)=x_{j}^{i}$ for all $i$ and $j<n$ then $f_{0}, \cdots, f_{n-1} \in \mathscr{F}_{0}^{1},\left\{x \mid f_{j}(x) \geqq k\right\}$ and $\left\{x \mid\left(f_{0}(x), \cdots, f_{n-1}(x)\right) \in R\right\}$ are co-finite, while $\left\{x \mid\left(f_{0}(x), \cdots, f_{n-1}(x)\right) \in R^{i}\right\}$ is not co-finite for $i<m$. Hence, $\mathscr{F}_{0}$ is good.

If $R, R^{0}, \cdots, R^{m-1}$ are recursive then $f_{0}, \cdots, f_{n-1}$ may clearly be defined recursively so that $\mathscr{F}_{\text {r }}$ is good. In each case $f_{0}, \cdots, f_{n-1}$ may be chosen such that they are in addition combinatorial (see the proof of Theorem 2 of Ellentuck (1967). Hence $\mathscr{F}_{c}$ and $\mathscr{F}_{r c}$ are also good.

A (possibly partial) function $g$ is recursively bounded if there is a recursive function $f$ such that $g(n) \leqq f(n)$ for every $n$ in the domain of $g$.

2.4.3. Let $\mathscr{F}_{1}$ be the set of recursively bounded functions in $\mathscr{F}_{0}$. Then $\mathscr{F}_{r} \subseteq \mathscr{F}_{1} \subseteq \mathscr{F}_{0}$. Let

$$
\mathscr{I}=\left\{f \in \mathscr{F}_{1}^{1} \mid \forall n n \leqq f(n)\right\} .
$$

LEMMA. $\mathscr{R}\left(\mathscr{F}_{1}\right)=$ The set of all relations and $\mathscr{F}_{1}$ is good. 
PRoOF. If $R \subseteq{ }^{n} \omega$ define $f, g \in \mathscr{F}_{1}^{n}$ as follows $f(x)=2\left(x_{0}+x_{1}+\cdots+x_{n-1}\right)$

$$
g(x)= \begin{cases}f(x) & \text { if } x \in R \\ f(x)+1 & \text { if } x \notin R,\end{cases}
$$

then $R=\left\{x \in \in^{n} \omega \mid f(x)=g(x)\right\}$. It only remains to show that $\mathscr{F}_{1}$ satisfies 2.3.1. Let $R, R^{0}, \cdots, R^{m-1} \subseteq{ }^{n} \omega$ such that $R \oiint_{e} R^{i}$ for all $i<m$. Let $\left\langle x^{i} \mid i<\omega\right\rangle$ be as in the proof of 2.4.2. Let $s_{j}=\max \left(x_{0}^{j}, \cdots, x_{n-1}^{j}\right)$. Define $f_{0}, \cdots, f_{n-1}$ as follows for $k<n$;

$$
f_{k}(x)= \begin{cases}0 & \text { if } x<s_{0} \\ x_{k}^{j} & \text { if } s_{j} \leqq x<s_{j+1}\end{cases}
$$

Then clearly each $f_{k}$ is nondecreasing and $f_{k}(x) \geqq x$ for all $x$, so that each $f_{k} \in \mathscr{F}_{1}^{1} . x \geqq s_{j}$ implies that $f_{k}(x) \geqq x_{k}^{j} \geqq j$. Hence $\left\{x \mid f_{k}(x) \geqq j\right\}$ is co-finite for all $j$. Also if $x \geqq s_{0}$ then $\left(f_{0}(x), \cdots, f_{n-1}(x)\right) \in R$ so that $\left\{x \mid\left(f_{0}(x), \cdots, f_{n-1}(x)\right) \in R\right.$ is co-finite. On the other hand $\left\{x \mid\left(f_{0}(x), \cdots, f_{n-1}(x)\right) \in R^{i}\right\}$ is not co-finite.

3.1. Definition. If $\mathscr{N}(Q, \mathscr{F})$ is an extension, $x$ is $\mathscr{N}(Q, \mathscr{F})$-universal if $x \in Q$ and for all $R \in \mathscr{R}^{1}(\mathscr{F}) x \in R_{Q}$ if and only if $R$ is co-finite.

This generalizes the notion of a universal isol. (See Ellentuck (1967).)

If $x$ is $\mathscr{N}(Q, \mathscr{F})$-universal let $Q[x]=\left\{f_{Q}(x) \mid f \in \mathscr{F}^{1}\right\}$. If $f \in \mathscr{F}^{n}$ let $f_{Q[x]}$ $=f_{Q} \wedge^{n} Q[x]$. If $R \in \mathscr{R}^{n}(\mathscr{F})$ let $R_{Q[x]}=R_{Q} \cap^{n} Q[x]$.

3.2. LemMa, If the extension $\mathscr{N}(Q, \mathscr{F})$ satisfies 1.4 .3 and $x$ is $\mathscr{N}(Q, \mathscr{F})$ universal then the mapping $F_{x}: \mathscr{F} / \sim \rightarrow Q[x]$ given by $F_{x}\left(f^{\sim}\right)=f_{Q}(x)$ for $f \in \mathscr{F}^{1}$ is a well-defined isomorphism of $\mathscr{N}^{\sim}(\mathscr{F})$ with $\mathscr{N}(Q[x], \mathscr{F})$.

Proof. To show that $F_{x}$ is well defined and one - one it is sufficient to show that $f^{\sim}=g^{\sim}$ if and only if $f_{Q}(x)=g_{Q}(x)$ for $f, g \in \mathscr{F}^{\prime}$, i.e. $R$ is cofinite if and only if $x \in R_{Q}$ where $R=\{y \mid f(y)=g(y)\}$ and using 1.4.3, $R_{Q}=\left\{y \in Q \mid f_{Q}(y)=g_{Q}(y)\right\}$. But this is just the definition of $\mathscr{N}(Q, \mathscr{F})$-universal.

To show that $F_{x}$ preserves the structure we need only consider functions as both $\mathscr{N}^{\sim}(\mathscr{F})$ and $\mathscr{N}(Q, \mathscr{F})$ satisfy 1.4.3., If $f \in \mathscr{F}^{n}$

$$
\begin{aligned}
F_{x}\left(f_{\mathscr{F} / \sim}\left(f_{0}^{\sim}, \cdots, f_{n-1}^{\sim}\right)\right) & =F_{x}\left(\left(f \circ\left(f_{0}, \cdots, f_{n-1}\right)\right)^{\sim}\right)=\left(f \circ\left(f_{0}, \cdots, f_{n-1}\right)\right)_{Q}(x) \\
& =f_{Q}\left(\left(f_{0}\right)_{Q}(x), \cdots,\left(f_{n-1}\right)_{Q}(x)\right)=f_{Q}\left(F_{x}\left(f_{0}^{\sim}\right), \cdots, F_{x}\left(f_{n-1}^{\sim}\right)\right) .
\end{aligned}
$$

3.3. CoRollary. If $\mathscr{F}$ is good and $\mathscr{N}(Q, \mathscr{F})$ is an extension satisfying 1.4.3 and there are $\mathscr{N}(Q, \mathscr{F})$-universal elements then $\mathscr{N}(Q, \mathscr{F})$ satisfies 1.4.6.

Proof. If $\mathscr{F}$ is good then $\mathscr{N}^{\sim}(\mathscr{F})$ satisfies 1.4.6. By $3.2 \mathscr{N}^{\sim}(\mathscr{F})$ is iso- 
morphically embeddable in $\mathscr{N}(Q, \mathscr{F})$. But clearly any extension of a system satisfying 1.4 .6 also satisfies 1.4 .6 . Hence $\mathscr{N}(Q, \mathscr{F})$ satisfies 1.4.6.

3.4. REMARK. The argument that Ellentuck (1967) outlines showing that $\mathscr{N}\left(\Lambda, \mathscr{F}_{r c}\right)$ is a Nerode extension may be formulated as an application of 1.4 and 3.3 .

\section{Part II}

4.1. If $\alpha \subseteq \omega$ let $\alpha(n)$ be the cardinality of $\{i \in \alpha \mid i<n\}$. Let $\alpha \leqslant \beta$ if there is a recursive function $f$ such that $\boldsymbol{\alpha}(n) \leqq \boldsymbol{\beta}(f(n))$ for all $n \in \omega$. Note that if such an $f$ exists then it may be taken to be in $\mathscr{I}$, that is, to be increasing, recursively bounded, and satisfy $n \leqq f(n)$. Let $\alpha \approx \beta$ if $\alpha \succcurlyeq \beta$ and $\beta \leqslant \alpha$. is a transitive relation so that $\approx$ is an equivalence relation. $D(\alpha)=\{\beta \mid \alpha \approx \beta\}$ is the recursive density type of $\alpha$. Let $\Delta=\{D(\alpha) \mid \alpha \subseteq \omega\}$. Let $D(\alpha) \leqq D(\beta)$ if $\alpha \leqq \beta$. Let $\infty=D(\omega)$. Identify $n \in \omega$ with $D(\{0, \cdots, n-1\})$. Then $\langle\Delta, \leqq\rangle$ is clearly a partial ordering with $\langle\omega$, $\leqq$ as initial segment and $\infty$ as last element.

4.2. We now give without proof some alternative characterizations of the relations $\preccurlyeq, \approx$ on sets of integers. Characterization (a) in each case shows that $\Delta$ is an analogue of the class of cardinal numbers in much the same way as $\Omega$, the set of R.E.T.'s.

If $\alpha \subseteq \omega$ is infinite let $\alpha_{0}, \alpha_{1}, \cdots$ enumerate $\alpha$ in order of magnitude.

4.2.1. LEMma. The following are equivalent to $\alpha \preccurlyeq \beta$.

(a) There is a one--one recursively bounded partial function defined on $\alpha$ and mapping $\alpha$ into $\beta$.

(b) There are $f_{1}, f_{2} \in \mathscr{I}$ such that $\alpha\left(f_{1}(n)\right) \leqq \beta\left(f_{2}(n)\right)$ for all $n$.

(c) $\alpha$ is finite and card $\alpha \leqq \operatorname{card} \beta$ or $\alpha, \beta$ are infinite and there is a recursive function $f$ such that $\beta_{n} \leqq f\left(\alpha_{n}\right)$ for all $n$.

4.2.2. The following are equivalent to $\alpha \approx \beta$.

(a) There is a one - one partial function $p$ such that $p$ and $p^{-1}$ are recursively bounded and $p$ has domain $\alpha$ and range $\beta$.

(b) There are $f_{1}, f_{2} \in \mathscr{I}$ such that

$$
\boldsymbol{\alpha} \circ f_{1}=\boldsymbol{\beta} \circ f_{2} \text {. }
$$

4.3. We shall define an extension $\mathscr{N}\left(\Delta, \mathscr{F}_{1}\right)$ on $\Delta$. If $f, g \in \mathscr{F}_{1}^{1}$ let $f \preccurlyeq g$ if $f(n) \leqq g(h(n))$ for all $n$, for some $h \in \mathscr{F}_{1}^{1}$.

Note in particular that $\alpha \preccurlyeq \beta$ if and only if $\alpha \leqslant \beta$.

4.3.1. Lemma. There is a well-defined mapping $D$ of $\mathscr{F}_{1} / \sim$ onto $\Delta$ such that $D\left(f_{1}^{\sim}\right) \leqq D\left(f_{2}^{\sim}\right)$ if and only if $f_{1} \preccurlyeq f_{2}$. 
Proof. If $f \in \mathscr{F}_{1}^{1}$ let $D\left(f^{\sim}\right)=D(\alpha)$ for an $\alpha \subseteq \omega$ such that $f=\alpha \circ g$ for some $g \in \mathscr{I}$. That such an $\alpha$ always exists is seen as follows. Let $g(n)=f(n)+n$. Then $g \in \mathscr{I}$, and if $\alpha=\omega-\{g(n+1)-1 \mid n \in \omega\}$ then $f=\alpha \circ g$. To see that $D\left(f^{\sim}\right)$ is well defined let $f^{\sim}=f_{1}^{\sim}, f=\alpha \circ g, f_{1}=\alpha_{1} \circ g_{1}$ for $\alpha, \alpha_{1} \subseteq \omega$, $g, g_{1} \in \mathscr{I}$. Then $\exists k \in \omega$ such that $\forall n \geqq k \alpha(g(n))=\alpha_{1}\left(g_{1}(n)\right)$. Let $g^{\prime}(n)=g(n+k)$, $g_{1}^{\prime}(n)=g_{1}(n+k)$. Then $g^{\prime}, g_{1}^{\prime} \in \mathscr{I}$ and $\alpha \circ g^{\prime}=\alpha_{1} \circ g_{1}^{\prime}$. Hence by 4.2 .2 (b) $D(\alpha)=D\left(\alpha_{1}\right)$. If $\alpha \subseteq \omega$ then $\alpha \in \mathscr{F}_{1}^{1}$ and $D\left(\alpha^{\sim}\right)=D(\alpha)$. Hence $D$ maps $\left.\mathscr{F}\right|_{1} \sim$ onto $\Delta$.

Let $f_{1}=\alpha_{1} \circ g_{1}, f_{2}=\alpha_{2} \circ g_{2}$. We wish to show that $f_{1} \preccurlyeq f_{2}$ if and only if $\alpha_{1} \preccurlyeq \alpha_{2}$. Let $f_{1}(n) \leqq f_{2}(h(n))$ for all $n$ where $h \in \mathscr{F}_{1}^{1}$. We may assume $h \in \mathscr{I}$. Then $\alpha_{1}\left(g_{1}(n)\right) \leqq \alpha_{2}\left(\left(g_{2} \circ h\right)(n)\right)$. But $g_{2} \circ h \in \mathscr{I}$ so that by 4.2 .1 (b) $\alpha_{1} \preccurlyeq \alpha_{2}$. Conversely, if $\alpha_{1}(n) \leqq \alpha_{2}(h(n))$ for all $n$ where $h \in \mathscr{I}$, then $\alpha_{1}(n) \leqq \alpha_{2}\left(g_{2}(h(n))\right)$ for all $n$, so

$$
f_{1}(n)=\alpha_{1}\left(g_{1}(n)\right) \leqq \alpha_{2}\left(g_{2}\left(h\left(g_{1}(n)\right)\right)\right)=f_{2}\left(h \circ g_{1}\right)(n) .
$$

Hence $f_{1} \preccurlyeq f_{2}$ as $h \circ g_{1} \in \mathscr{F}_{1}^{1}$.

4.3.2. If $f \in \mathscr{F}_{1}^{n}$ let $f_{\Delta}\left(D\left(f_{0}^{\sim}\right), \cdots, D\left(f_{n-1}^{\sim}\right)\right)=D\left(\left(f \circ\left(f_{0}, \cdots, f_{n-1}\right)\right) \sim\right)$. This is well defined, for if $f_{i} \preccurlyeq g_{i}$ for $i<n$ then $f \circ\left(f_{0}, \cdots, f_{n-1}\right) \preccurlyeq g \circ\left(g_{0}, \cdots, g_{n-1}\right)$.

If $R \subseteq{ }^{n} \omega$ let $\left.R_{\Delta}=\left\{\left(D\left(f_{0}^{\sim}\right), \cdots, D\left(f_{n-1}^{\sim}\right)\right) \mid f_{0}^{\sim}, \cdots, f_{n-1}^{\sim}\right) \in R_{\mathscr{F}_{1} / \sim}\right\}$. Clearly

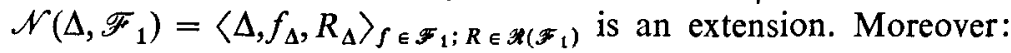

4.3.3. $D: \mathscr{N}^{\sim}\left(\mathscr{F}_{1}\right) \rightarrow \mathscr{N}\left(\Delta, \mathscr{F}_{1}\right)$ is a homomorphism. Note that if $R=\left\{(x, y) \epsilon^{2} \omega \mid x=y\right\}$ and $S=\left\{(x, y) \in^{2} \omega \mid x \leqq y\right\}$ then $R_{\Delta}=\{(x, y) \in$ $\left.{ }^{2} \Delta \mid x=y\right\}$ and $S_{\Delta}=\left\{(x, y) \in{ }^{2} \Delta \mid x \leqq y\right\}$.

4.3.4. Lemma. Let $\phi$ be a sentence of $\mathscr{L}\left(\mathscr{F}_{1}\right)$ of the form $\forall v_{0}, \cdots, v_{k-1}$ $\left[R\left(v_{0}, \cdots, v_{k-1}\right) \rightarrow \psi\right]$ where $\psi$ is atomic. If $\mathcal{N}\left(\mathscr{F}_{1}\right) \vDash \phi$ then $\mathcal{N}\left(\Delta, \mathscr{F}_{1}\right) \vDash \phi$.

Proof. As $\mathscr{N}^{\sim}\left(\mathscr{F}_{1}\right)$ is a Nerode extension, $R\left(v_{0}, \cdots, v_{k-1}\right) \rightarrow \psi$ is a Horn reduct of itself and $\mathscr{N}\left(\mathscr{F}_{1}\right) \vDash \phi$ it follows that $\mathscr{N}^{\sim}\left(\mathscr{F}_{1}\right) \vDash \phi$. But it is easy to see that sentences of the form $\phi$ are preserved under homomorphic images. Hence by $4.3 .3 \mathscr{N}\left(\Delta, \mathscr{F}_{1}\right) \vDash \phi$.

4.3.5. Corollary. $\mathscr{N}\left(\Delta, \mathscr{F}_{1}\right)$ satisfies $1.4 .1,1.4 .2,1.4 .3^{\prime}, 1.4 .4^{\prime}$ and 1.4 .5 , where

1.4.3'. If $R \subseteq\left\{x \in{ }^{n} \omega \mid f(x)=g(x)\right\}$ for $f, g \in \mathscr{F}^{n}$ and $R \in \mathscr{R}^{n}(\mathscr{F})$ then $R_{Q} \subseteq\left\{x \in{ }^{n} Q \mid f_{Q}(x)=g_{Q}(x)\right\}$.

1.4.4'. If $R, S \in \mathscr{R}^{n}(\mathscr{F})$ then $(R \cap S)_{Q} \subseteq R_{Q} \cap S_{Q}$.

4.4. The definition 4.4 .1 of the set $\Gamma$ of recursive density types enables us to give a direct proof of 4.4.2. Later we shall give a simple characterization of $\Gamma$ in terms of the partial ordering on $\Delta$. 
4.4.1. Definition. Let $\mathscr{C}=\{\alpha \mid(\forall f \in \mathscr{I})(\exists g \in \mathscr{I}) \alpha \circ g=\alpha \circ f \circ g\}$.

$$
\Gamma=\{D(\alpha) \mid \alpha \in \mathscr{C}\} \text {. }
$$

4.4.2. THEOREM. Let $k \leqq l \leqq n, m \in \omega$. Let $f^{i} \in \mathscr{F}_{1}^{l}, g^{i} \in \mathscr{F}^{k+n-l}$ for $i<m$. Let $\psi$ be an atomic formula of $\mathscr{L}\left(\mathscr{F}_{1}\right)$ containing variables from $v_{0}, \cdots, v_{n-1}$. Let $\phi$ be the formula

$$
\left(\wedge\left[\underline{f}^{i}\left(v_{0}, \cdots, v_{l-1}\right) \leqq \underline{g}^{i}\left(v_{0}, \cdots, v_{k-1}, v_{l}, \cdots, v_{n-1}\right)\right] \rightarrow \psi\right) .
$$

If $\mathscr{N}\left(\mathscr{F}_{1}\right) \vDash \forall v_{0}, \cdots, \forall v_{n-1} \phi$ then $\mathscr{N}\left(\Delta, \mathscr{F}_{1}\right) \vDash \phi[x]$ for all $x \in{ }^{n} \Delta$ such that $x_{i} \in \Gamma$ for $i<k$.

Proof. By 4.3.4 and 2.4.3 we assume without loss of generality that $\psi$ has the form $\underline{R}\left(v_{0}, \cdots, v_{n-1}\right)$. Assume that $\mathscr{N}\left(\mathscr{F}_{1}\right) \vDash \forall v_{0}, \cdots, \forall v_{n-1} \phi$, that $x \in \in^{n} \Delta$ such that $x_{i} \in \Gamma$ for $i<k$ and that

$$
f_{\Delta}^{i}\left(x_{0}, \cdots, x_{l-1}\right) \leqq g_{\Delta}^{i}\left(x_{0}, \cdots, x_{k-1}, x_{l}, \cdots, x_{n-1}\right) \text { for all } i<m .
$$

Let $D\left(\alpha_{i}\right)=x_{i}$ for $i<n$, such that $\alpha_{i} \in \mathscr{C}$ for $i<k$. To prove the theorem we must show that $x \in R_{\Delta}$.

By $\left(^{*}\right) f^{i} \circ\left(\alpha_{0}, \cdots, \alpha_{l-1}\right) \preccurlyeq g^{i} \circ\left(\alpha_{0}, \cdots, \alpha_{k-1}, \alpha_{l}, \cdots, \alpha_{n-1}\right)$ for all $i<m$, i.e. there are functions $h^{i} \in \mathscr{I}$ for $i<m$ such that for all $y \in \omega$

$$
\begin{aligned}
f^{i}\left(\boldsymbol{\alpha}_{0}(y), \cdots, \boldsymbol{\alpha}_{l-1}(y)\right) & \leqq g^{i}\left(\boldsymbol{\alpha}_{0}\left(h^{i}(y)\right), \cdots, \boldsymbol{\alpha}_{k-1}\left(h^{i}(y)\right), \boldsymbol{\alpha}_{l}\left(h^{i}(y)\right), \cdots, \boldsymbol{\alpha}_{n-1}\left(h^{i}(y)\right)\right) \\
& \leqq g^{i}\left(\boldsymbol{\alpha}_{0}(h(y)), \cdots, \boldsymbol{\alpha}_{k-1}(h(y)), \boldsymbol{\alpha}_{l}(h(y)), \cdots, \boldsymbol{\alpha}_{n-1}(h(y))\right)
\end{aligned}
$$

where $h(y)=\operatorname{Max}\left\{h^{i}(y) \mid i<m\right\}$. Then $h \in \mathscr{I}$. Define $j_{0}, \cdots, j_{k-1} \in \mathscr{I}$ to satisfy the following conditions,

$$
\begin{aligned}
\alpha_{0} \circ j_{0}= & \alpha_{0} \circ h \circ j_{0} \\
\alpha_{1} \circ j_{1}= & \alpha_{1} \circ\left(h \circ j_{0}\right) \circ j_{1} \\
& \cdots \\
\alpha_{k-1} \circ j_{k-1}= & \alpha_{k-1} \circ\left(h \circ j_{0} \circ \cdots \circ j_{k-2}\right) \circ j_{k-1} .
\end{aligned}
$$

This is possible as $\alpha_{0}, \cdots, \alpha_{k-1} \in \mathscr{C}$ and $h, h \circ j_{0}, \cdots,\left(h \circ j_{0} \circ \cdots \circ j_{k-2}\right) \in \mathscr{I}$. Let $j=j_{0} \circ j_{1} \circ \cdots \circ j_{k-1} \in \mathscr{I}$. Then clearly $\alpha_{0} \circ j=\alpha_{0} \circ h \circ j, \cdots, \alpha_{k-1} \circ j=\alpha_{k-1} \circ h \circ j$. Hence, for all $y \in \omega$

$f^{i}\left(\boldsymbol{\alpha}_{0}(j(y)), \cdots, \boldsymbol{\alpha}_{l-1}(j(y))\right) \leqq g^{i}\left(\boldsymbol{\alpha}_{0}(j(y)), \cdots, \boldsymbol{\alpha}_{k-1}(j(y)), \boldsymbol{\alpha}_{l}(h(j(y))), \cdots, \boldsymbol{\alpha}_{n-1}(h(j(y)))\right)$.

Thus, by hypothesis,

$$
\left(\boldsymbol{\alpha}_{0}(j(y)), \cdots, \boldsymbol{\alpha}_{l-1}(j(y)), \boldsymbol{\alpha}_{l}(h(j(y))), \cdots, \boldsymbol{\alpha}_{n-1}(h(j(y)))\right) \in R
$$

for all $y$. But $j, h \circ j \in \mathscr{I}$ so that 


$$
\begin{aligned}
D\left(\left(\alpha_{i} \circ j\right)^{\sim}\right) & =D\left(\alpha_{i}\right) \text { for } i<l \\
D\left(\left(\alpha_{i} \circ h \circ j\right)^{\sim}\right) & =D\left(\alpha_{i}\right) \text { for } l \leqq i<n
\end{aligned}
$$

and $x=\left(D\left(\alpha_{0}\right), \cdots, D\left(\alpha_{n-1}\right)\right) \in R_{\Delta}$.

4.5. Before investigating $\Gamma$ we shall derive some consequences from the following special case of 4.4 .2 that does not involve $\Gamma$.

4.5.1. Let $\phi$ be as in 4.4 .2 with $k=0$. Then $\mathscr{N}\left(\mathscr{F}_{1}\right) \vDash \forall v_{0} \cdots v_{n-1} \phi$ implies that $\mathscr{N}\left(\Delta, \mathscr{F}_{1}\right) \vDash \forall v_{0} \cdots v_{n-1} \phi$.

4.5.2. Let $f(x, y)=\max (x, y), g(x, y)=\min (x, y)$, then $f, g \in \mathscr{F}_{1}^{2}$ and $\langle\Delta, \leqq, f, g\rangle$ is a distributive lattice. 4.5.1 applies to each of the axioms of the theory of a distributive lattice; so that $\left\langle\Delta, \leqq, f_{\Delta}, g_{\Delta}\right\rangle$ is a distributive lattice. Let $x \cup y=f_{\Delta}(x, y) x \cap y=g_{\Delta}(x, y)$ for $x, y \in \Delta$.

4.5.3. If $f \in \mathscr{F}_{1}^{1}$ is one - one then

$$
f(x) \leqq f(y) \leftrightarrow x \leqq y \text { for } x, y \in \omega .
$$

Hence by 4.5.1 $f_{\Delta}(x) \leqq f_{\Delta}(y) \leftrightarrow x \leqq y$ for $x, y \in \Delta$, and $f_{\Delta}$ is one - one.

If $f \in \mathscr{I}$ then by $4.5 .1 x \leqq f_{\Delta}(x)$ for all $x \in \Delta$.

4.5.4. If $f(x, y)=x+y$ and $g(x, y)=x \cdot y$ for $x, y \in \omega$ then $f, g \in \mathscr{F}_{1}^{2}$. Let $x+y=f_{\Delta}(x, y), x \cdot y=g_{\Delta}(x, y)$ for $x, y \in \Delta$. It is not hard to show that these operations are the same as those introduced in Gonsher and Rice (1969), i.e. that $D(\alpha)+D(\beta)=D(\alpha \cup \beta)$ if $\alpha, \beta \subseteq \omega$ and $\alpha \cap \beta=\phi D(\alpha) \cdot D(\beta)$ $=D(\{j(x, y) \mid x \in \alpha$ and $y \in \beta\})$, where $j:{ }^{2} \omega-\omega$ is a one-one recursive function. Observe that

4.5.5. $x+1=x$ if and only ig $x=\infty$. (See Gonsher and Rice 1969).) In Gonsher and Rice (1969) a pair of disjoint sets $\alpha, \beta$ are constructed such that $D(\alpha)<\infty, D(\beta)<\infty$ but $D(\alpha)+D(\beta)=D(\alpha \cup \beta)=\infty$. Hence $\Lambda-\{\infty\}$ is not closed under every $f_{\Delta}$ for $f \in \mathscr{F}_{1}$. On the other hand we have

4.5.6. Lemma. If $f \in \mathscr{F}_{1}^{n}$ and $x \in{ }^{n} \Delta$ then $x_{0} \cup x_{1} \cup \cdots \cup x_{n-1}<\infty$ implies $f_{\Delta}(x)<\infty$.

Proof. If $f \in \mathscr{F}_{1}^{n}$ then there is a $g \in \mathscr{I}$ such that

$$
f(x) \leqq g\left(\max \left(x_{0}, \cdots, x_{n-1}\right)\right) \text { for } x \in{ }^{n} \omega .
$$

By 4.5.1 $f_{\Delta}(x) \leqq g_{\Delta}\left(x_{0} \cup \cdots \cup x_{n-1}\right)$ for $x \in{ }^{n} \Delta$. We may assume that $g$ is one one so that $x \leqq g_{\Delta}(x) \leqq g_{\Delta}(y)$ implies $x \leqq y$ for $x, y \in{ }^{n} \Delta$. Hence $\infty \leqq g_{\Delta}(y)$ implies $\infty \leqq y$.

Hence $\infty \leqq f_{\Delta}(x)$ implies $\infty \leqq g_{\Delta}\left(x_{0} \cup \cdots \cup x_{n-1}\right)$, which implies that $\infty \leqq x_{0} \cup \cdots \cup x_{n-1}$; i.e. $f_{\Delta}(x)=\infty$ implies $x_{0} \cup \cdots \cup x_{n}=\infty$. Taking the contrapositive gives the lemma. 
4.5.7. CoRollary. $\Delta-\{\infty\}$ is closed under every $f_{\Delta}$ for $f \in \mathscr{F}_{1}^{1}$.

4.6. In 4.6.2 we give a simple characterization of $\Gamma$.

4.6.1. $D(\alpha)=\infty$ if and only if there is an $h \in \mathscr{I}$ such that $h(n) \in \alpha$ for all $n$.

Proof. If $D(\alpha)=\infty$ then there is a $g \in \mathscr{I}$ such that $\alpha(g(n)) \geqq n$ for all $n$. Let

$$
\left\{\begin{array}{l}
g^{\prime}(0)=0 \\
g^{\prime}(n+1)=g\left(g^{\prime}(n)+1\right) .
\end{array}\right.
$$

Then $g^{\prime} \in \mathscr{I}$ and $\alpha\left(g^{\prime}(n+1)\right) \geqq g^{\prime}(n)+1$. But $\alpha\left(g^{\prime}(n)\right) \leqq g^{\prime}(n)$. So $(\exists y \in \alpha) g^{\prime}(n)$ $\leqq y<g^{\prime}(n+1)$. Let $h(n)$ be such a $y$ for all $n$. Then $h \in \mathscr{I}$ and $h(n) \in \alpha$ for all $n$.

Conversely, let $h(n) \in \alpha$ for all $n$ where $h \in \mathscr{I}$. Let

$$
\left\{\begin{array}{l}
g(0)=0 \\
g(n)+1=h(g(n)+1) .
\end{array}\right.
$$

Then $g \in \mathscr{I}$ and $g(n) \in \alpha$ for all $n$. Also $g(n+1)>g(n)$ for all $n$, so that $\alpha(g(n)) \geqq n$. Hence $D(\alpha)=\infty$.

4.6.2. Lemma. $\Gamma=\{x \in \Delta \mid(\forall y<\infty) x \cup y<\infty\}$.

Proof. If $x \in \Gamma$ then by 4.4 .2 ,

$$
x+z \leqq x+y \text { implies } z \leqq y \text { for all } z, y \in \Delta .
$$

Let $z=\infty$, so that $\infty \leqq x+y$ implies $\infty \leqq y$. Hence $y<\infty$ implies $x \cup y$ $\leqq x+y<\infty$. So $\Gamma \subseteq\{x \in \Delta \mid(\forall y<\infty) x \cup y<\infty\}$.

Conversely, suppose $(\forall y<\infty) x \cup y<\infty$. Let $x=D(\alpha)$ and $f \in \mathscr{I}$. We must find $g \in \mathscr{I}$ such that $\alpha \circ g=\alpha \circ f \circ g$. Let $\beta=\{n \mid \alpha(n)=\alpha(f(n)+1)\}$. Clearly $\alpha \cap \beta=\varnothing$. Let

$$
\left\{\begin{array}{l}
g^{\prime}(0)=0 \\
g^{\prime}(n+1)=f\left(g^{\prime}(n)\right)+1 .
\end{array}\right.
$$

Then $g^{\prime} \in \mathscr{I}$ and for all $n \in \omega$ there is a $y \in \alpha \cup \beta$ such that $g^{\prime}(n) \leqq y<g^{\prime}(n+1)$. Hence $n \leqq(\boldsymbol{\alpha} \cup \boldsymbol{\beta})\left(g^{\prime}(n)\right)$ for all $n$, so that $D(\alpha \cup \beta)=\infty$. So $x+D(\beta)$ $=D(\alpha \cup \beta)=\infty$, and by $4.5 .6 \times \cup D(\beta)=\infty$, hence $D(\beta)=\infty$, i.e., by 4.6 .1 there is an $h \in \mathscr{I}$ such that $h(n) \in \beta$ for all $n$; i.e. $\alpha(h(n))=\alpha(f(h(n))+1)$ for all $n$ by definition of $\beta$. Hence $\alpha \circ h=\alpha \circ f \circ h$, and $\alpha \in \mathscr{C}$. Note that the proof has shown that $D(\alpha) \in \Gamma$ if and only if $\alpha \in \mathscr{C}$.

4.6.3. Corollary. (i) $x_{1} \leqq x_{2} \in \Gamma$ implies $x_{1} \in \Gamma$. (ii) If $f \in \mathscr{F}_{1}^{n}$ and $x \in \in^{n} \Gamma$ then $f_{\Delta}(x) \in \Gamma$. 
Proof. (i) If $(\forall y<\infty) x_{2} \cup y<\infty$ and $x_{1} \leqq x_{2}$ then $(\forall y<\infty) x_{1} \cup y$ $\leqq x_{2} \cup y<\infty$.

(ii) If $x \in{ }^{n} \Gamma$ and $y<\infty$ then $x_{n-1} \cup y<\infty$ so $x_{n-2} \cup\left(x_{n-1} \cup y\right)<\infty \cdots$. Hence $x_{0} \cup x_{1} \cup \cdots \cup x_{n-1} \cup y<\infty$. Let $g\left(x_{0}, \cdots, x_{n-1}, x_{n}\right)=f(x) \cup x_{n}$ for $x_{0}, \cdots, x_{n} \in \omega$. Hence

$$
g_{\Delta}\left(x_{0}, \cdots, x_{n+1}, y\right)=f_{\Delta}(x) \cup y .
$$

By 4.5.6 and the above, $g_{\Delta}\left(x_{0}, \cdots, x_{n+1}, y\right)<\infty$; i.e., we have shown that if $x \in{ }^{n} \Gamma$ then $\forall y<\infty f_{\Delta}(x) \cup y<\infty$, i.e. $f_{\Delta}(x) \in \Gamma$.

4.6.4. Definition. If $f \in \mathscr{F}_{1}^{n}$ let $f_{\Gamma}=f_{\Delta} \Gamma^{n} \Gamma$. If $R \subseteq \omega$, let $R_{\Gamma}=R_{\Delta} \cap{ }^{n} \Gamma$. Then $\mathscr{N}\left(\Gamma, \mathscr{F}_{1}\right)$ is an extension.

4.7. The proof of the next theorem uses the existence of $\mathscr{N}\left(\Gamma, \mathscr{F}_{1}\right)$-universal elements. This will be proved in 4.8 .

THEOREM. $\mathscr{N}\left(\Gamma, \mathscr{F}_{1}\right)$ is a Nerode extension.

Proof. By 3.3, 1.4 and 2.4.3 it is sufficient to show that $\mathscr{N}\left(\Gamma, \mathscr{F}_{1}\right)$ satisfies 1.4.1-1.4.5. By 4.3.5 $\mathscr{N}\left(\Delta, \mathscr{F}_{1}\right)$ satisfies $1.4 .1,1.4 .2,1.4 .3^{\prime}, 1.4 .4^{\prime}$ and 1.4 .5 and hence so does $\mathscr{N}\left(\Gamma, \mathscr{F}_{1}\right)$. To finish the proof it remains only to show 4.7.1 and 4.7.2.

4.7.1. If $\left\{x \in{ }^{k} \omega \mid f(x)=g(x)\right\} \subseteq R$ for $f, g \in \mathscr{F}_{1}^{k}$ then $\left\{x \in{ }^{k} \Gamma \mid f_{\Gamma}(x)\right.$ $\left.=g_{\Gamma}(x)\right\} \subseteq R_{\Gamma}$.

4.7.2. If $R, S \subseteq{ }^{k} \omega$ then $R_{\Gamma} \cap S_{\Gamma} \subseteq(R \cap S)_{\Gamma}$. Using 4.4 .2 with $k=l=n$, we get 4.7.1 where $\phi$ is the formula

$$
\left[\underline{f}\left(v_{0}, \cdots, v_{k-1}\right) \leqq \underline{g}\left(v_{0}, \cdots, v_{k-1}\right) \cup \underline{g}\left(v_{0}, \cdots, v_{k-1}\right) \leqq \underline{f}\left(v_{0}, \cdots, v_{k-1}\right)\right] \rightarrow \underline{R}\left(v_{0}, \cdots, v_{k-1}\right) .
$$

To show 4.7.2, let $R=\left\{x \in{ }^{k} \omega \mid f(x)=g(x)\right\}$ and $S=\left\{x \in{ }^{k} \omega \mid f^{\prime}(x)=g^{\prime}(x)\right\}$ for $f, g, f^{\prime}, g^{\prime} \in \mathscr{F}_{1}^{k}$. Then by $1.4 .3^{\prime}$ for $\mathscr{N}\left(\Gamma, \mathscr{F}_{1}\right)$

$$
R_{\Gamma} \cap S_{\Gamma} \subseteq\left\{x \in{ }^{k} \Gamma \mid f_{\Gamma}(x)=g_{\Gamma}(x) \wedge f_{\Gamma}^{\prime}(x)=g_{\Gamma}^{\prime}(x)\right\} .
$$

By another application of 4.4.2 $f_{\Gamma}(x)=g_{\Gamma}(x)$ and $f_{\Gamma}^{\prime}(x)=g_{\Gamma}^{\prime}(x)$ implies $x \in(R \cap S)_{\Gamma}$ for all $x \in{ }^{k} \Gamma$. Hence $R_{\Gamma} \cap S_{\Gamma} \subseteq(R \cap S)_{\Gamma}$.

4.8. By one of the standard definitions of the hyperimmune sets, $\alpha$ is hyperimmune if and only if $D(\alpha) \in \Delta-(\omega \cup\{\infty\})$. Hence the hyperimmune sets form an invariant of the recursive density types. Another example is the class $\mathscr{C}$. We shall examine a few more such invariants and finally prove the existence of $\mathscr{N}\left(\Gamma, \mathscr{F}_{1}\right)$-universal elements. The following definitions are in Gonshor and Rice (1969).

4.8.1. The infinite set $\alpha$ is strongly hyperimmune (s.h.) if $\left\{n \mid \alpha_{n}>h(n)\right\}$ is co-finite for every recursive function $h$. 
4.8.2. The infinite set $\alpha$ is uniformly hyperimmune (u.h.) if $\left\{n \mid \alpha_{n+1}>h\left(\alpha_{n}\right)\right\}$ is co-finite for every recursive function $h$.

It is not hard to show the following.

4.8.3. $\alpha$ is s.h. (u.h.) if and only if $\alpha$ is infinite and $\{n \mid \alpha(h(n)) \leqq n\}$ $(\{n \mid \alpha(h(n)) \leqq \alpha(n)+1\}$ is co-finite for all $h \in \mathscr{I}$.

4.8.4. Definition. $\alpha$ is universal if $\alpha$ is infinite and $\{n \mid \alpha(h(n+1))$ $\leqq \alpha(h(n))+1\}$ is co-finite for all $h \in \mathscr{I}$.

In Gonshor and Rice (1969) it is shown that the classes of s.h. and u.h. sets are invariants of the recursive density types. Let

$$
\begin{aligned}
& \Delta_{s}=\{D(\alpha) \mid \alpha \text { is finite or } \alpha \text { is s.h. }\} \\
& \Delta_{u}=\{D(\alpha) \mid \alpha \text { is finite or } \alpha \text { is u.h. }\}
\end{aligned}
$$

Then $\Delta_{u} \subseteq \Delta_{s} \subseteq \Gamma$ where all the inclusions are proper, $\Delta_{s}$ is an ideal of the lattice $\langle\Delta, \leqq\rangle$ and $\Delta_{u}=\left\{A \in \Delta \mid\left(\forall B \in \Delta^{\infty}\right)(\forall C \in \Delta) A \neq 2 B+C\right\}$; (see Gonshor and Rice (1969)).

4.8.5. LeMmA. $\alpha$ is universal if and only if $D(\alpha)$ is $\mathscr{N}\left(\Delta, \mathscr{F}_{1}\right)$-universal.

Proof. $D(\alpha)$ is $\mathscr{N}\left(\Delta, \mathscr{F}_{1}\right)$-universal if and only if whenever $\alpha(h(n)) \in R$ for all $n$, where $h \in \mathscr{I}$, then $R$ is co-finite. This is true if and only if the range of $\alpha \circ h$ is co-finite for all $h \in \mathscr{I}$. But the range of $\alpha \circ h$ is co-finite if and only if $\{n \mid \alpha(h(n+1)) \leqq \alpha(h(n))+1\}$ is co-finite, concluding the proof.

4.8.6. Lemma. If $X$ is a countable family of infinite sets of integers then there is a u.h. set $\alpha$ such that $\beta$ is not $\preccurlyeq \alpha$ for all $\beta \in X$.

PROOF. Let $\left\langle h^{i} \mid i \in \omega\right\rangle$ be a sequence of strictly increasing functions such that $x<h^{i}(x)<h^{i+1}(x)$ for all $i, x \in \omega$ and if $h$ is recursive then $\forall x h(x) \leqq h^{i}(x)$ for some $i \in \omega$. Such a sequence may easily be constructed as the set of recursive functions is countable. Let $\left\langle\alpha^{i} \mid i \in \omega\right\rangle$ be an enumeration of $X$ such that each set in $X$ occurs infinitely often in the enumeration. Now define $\alpha=\left\{\alpha_{0}<\alpha_{1}<\cdots\right\}$ as follows:

$$
\left\{\begin{aligned}
\alpha_{0} & =h^{0}\left(\alpha_{0}^{0}\right) \\
\alpha_{n+1} & =h^{n+1}\left(\alpha_{\alpha_{n}+1}^{n+1}\right) .
\end{aligned}\right.
$$

Then $\alpha$ is u.h., for if $h$ is recursive and $h(x) \leqq h^{i}(x)$ for all $x$ then

$$
\alpha_{n+1}=h^{n+1}\left(\alpha_{\alpha_{n}+1}^{n+1}\right) \geqq h^{n+1}\left(\alpha_{n}\right)>h\left(\alpha_{n}\right)
$$

for all $n \geqq i$.

Finally, if $\beta \in X$ to show that $\beta \preccurlyeq \alpha$; by 4.2 .1 (c) it is sufficient to show that for all $n \exists x \alpha_{x}>h^{n}\left(\beta_{x}\right)$. Given $n$ there is an $m \geqq n$ such that $\beta=\alpha^{m+1}$. Then 


$$
\alpha_{m+1}=h^{m+1}\left(\begin{array}{c}
m+1 \\
\alpha_{\alpha_{m+1}}
\end{array}\right)>h^{n}\left(\alpha_{m+1}^{m+1}\right)=h^{n}\left(\beta_{m+1}\right) .
$$

Hence $\alpha_{x}>h^{n}\left(\beta_{x}\right)$ with $x=m+1$.

4.8.7. COROLlary. There are uncountably many elements of $\Gamma$ that are $\mathscr{N}\left(\Gamma, \mathscr{F}_{1}\right)$-universal.

Proof. By 4.8.3, 4.8.4 and 4.8.5 every $x \in \Delta_{u}-\omega$ is $\mathscr{N}\left(\Delta, \mathscr{F}_{1}\right)$-universal. But as $\Delta_{u} \subseteq \Gamma$, every $x \in \Delta_{u}-\omega$ is $\mathscr{N}\left(\Gamma, \mathscr{F}_{1}\right)$-universal. By 4.8.6 $\Delta_{u}$ is uncountable.

4.8.8. Lemma. If $f \in \mathscr{F}_{1}^{n}$ and $x \in{ }^{n} \Delta_{s}$ then $f_{\Delta}(x) \in \Delta_{s}$.

Proof. If $f \in \mathscr{F}_{1}^{n}$ then there is a $g \in \mathscr{I}$ such that $f(x) \leqq g\left(\max \left\{x_{i} \mid i<n\right\}\right)$ for $x \in{ }^{n} \omega$. Hence, as $\Delta_{s}$ is an ideal $x \in{ }^{n} \Delta_{s}$ implies $y=x_{0} \cup \cdots \cup x_{n-1} \in \Delta_{s}$ and as $f_{\Delta}(x) \leqq g_{\Delta}(y), f_{\Delta}(x) \in \Delta_{s}$ if

$$
\text { for all } y \in \Delta_{s}, g_{\Delta}(y) \in \Delta_{s} \text {. }
$$

To prove (**) let $y=D(\alpha) \in \Delta_{s}$ so that $\alpha$ is s.h. We must show that $D((g \circ \alpha) \sim) \in \Delta_{s}$. By the proof of 4.3.1 $g \circ \alpha=\beta \circ g^{\prime}$ for some $\beta \subseteq \omega$ and $g^{\prime} \in \mathscr{I}$. Hence we must show that $\beta$ is s.h. Let $h \in \mathscr{I}$. We show that $\{n \mid \boldsymbol{\beta}(h(n)) \leqq n\}$ is co-finite.

$$
\boldsymbol{\beta}(h(g(n+1))) \leqq \boldsymbol{\beta} \circ g^{\prime}(h(g(n+1)))=g(\boldsymbol{\alpha}(h(g(n+1)))) .
$$

As $\alpha$ is s.h. $\{n \mid \alpha(h(g(n+1))) \leqq n\}$ is co-finite. So $\{n \mid \beta(h(g(n+1))) \leqq g(n)\}$ is co-finite,

$$
\text { i.e. } \exists m \forall n \geqq m \boldsymbol{\beta}(h(g(n+1))) \leqq g(n) .
$$

If $x \geqq g(m)$ then $\exists n \geqq m g(n) \leqq x \leqq g(n+1)$. So $\boldsymbol{\beta}(h(x)) \leqq \boldsymbol{\beta}(h(g(n+1)))$ $\leqq g(n) \leqq x$; i.e. $\{x \mid \boldsymbol{\beta}(h(x)) \leqq x\}$ is co-finite.

4.8.9. By 4.8.8 we may define in the obvious way the extension $\mathscr{N}\left(\Delta_{s}, \mathscr{F}_{1}\right)$ as a subsystem of $\mathscr{N}\left(\Gamma, \mathscr{F}_{1}\right)$. As $\Delta_{u} \subseteq \Delta_{s}$ there are uncountably many $\mathscr{N}\left(\Delta_{s}, \mathscr{F}_{1}\right)$ universal elements. As $\mathscr{N}\left(\Gamma, \mathscr{F}_{1}\right)$ satisfies 1.4.1-1.4.5, so does $\mathscr{N}\left(\Delta_{s}, \mathscr{F}_{1}\right)$. Hence we have

THEOREM. $\mathscr{N}\left(\Delta_{s}, \mathscr{F}_{1}\right)$ is a Nerode extension.

\section{References}

E. Ellentuck (1967), 'Universal isols', Math. Z. 98, 1-8.

H. Gonshor and H. Rice (1969), 'Recursive density types', Trans. Amer. Math. Soc. 140, 493-503.

A. Nerode (1961), 'Extensions to isols', Ann. of Math. 73, 362-403.

Pavlova (1961), Soviet Math. Dokl. 2, 1017-1019.

Department of Mathematics

Manchester University

England 